


\title{
Growth of oriented rare-earth-transition-metal thin films
}

\author{
Eric E. Fullerton ${ }^{1}$, C. H. Sowers ${ }^{1}$, X.Z. Wu ${ }^{1,2}$, and S. D. Bader ${ }^{1}$ \\ 'Materials Science Division, Argonne National Laboratory, Argonne, IL 60439 \\ ${ }^{2}$ Department of Physics, Northern Illinois University, De Kalb, IL 60115
}

\begin{abstract}
Rare-earth-transitionmetal thin films are successfully grown by magnetron sputtering onto single-crystal MgO substrates with epitaxial $W$ buffer layers. The use of epitaxial $W$ buffer layers allows oriented single-phase films to be grown. Sm-Co films grown onto W(100), have strong in-plane anisotropy and coercivities exceeding $5 \mathrm{~T}$ at $5 \mathrm{~K}$ whereas Fe-Sm films have strong perpendicular anisotropy and are magnetically soft.
\end{abstract}

\section{Introduction}

Growth of epitaxial transition-metal superlattices has proven essential in elucidating the role of crystal orientation and structure on magnetic properties such as giant magnetoresistance and interlayer coupling [1]. Extending these studies to the growth of epitaxial rare earth-transition metal (RE-TM) films and superlattices hold promise in further exploring and optimizing the properties of magnetic films and, in particular, those of hardmagnet films. For instance, Skomski and Coey predict that a giant energy product (120 MG Oe) is possible in multilayer structures consisting of aligned hard-magnet layers interleaved with soft-phase layers with high magnetization [2]. Experimental realization of such structures depends on the growth of epitaxial hard-magnet thin films. Epitaxial growth also allows the magnetic properties to be tailored by controlling the crystal orientation and the anisotropies of the magnetic layers and opens the possibility of synthesizing a range of RE-TM phases [3]. In the present work, we study the growth and magnetic properties of a variety of $\mathrm{RE}-\mathrm{TM}$ thin films $(\mathrm{RE}=\mathrm{Sm}$, $\mathrm{Nd}, \mathrm{TM}=\mathrm{Fe} ; \mathrm{Co}$ ) on epitaxial TM buffer layers.

\section{Synthesis and Characterization}

The 10-20 $\mathrm{nm}$ buffer layers ( $\mathrm{Cr}$ or $\mathrm{W}$ ) are deposited by magnetron sputtering onto singlecrystal $\mathrm{MgO}(100)$ and (111) substrates at a substrate

Manuscript received March 1, 1996

Work supported by US DOE-Basic Energy SciencesMaterials Science Contract \#W-31-109-ENG-38. 
temperature $T_{s}$ of $600{ }^{\circ} \mathrm{C}$. This results in (100) and (110) epitaxial growth onto $\mathrm{MgO}(100)$ and (111), respectively for both $\mathrm{Cr}$ and $\mathrm{W}[4]$. In this paper we concentrate on the films grown onto $\mathrm{W}$ buffer layers. The RE-TM films are deposited by co-sputtering from elemental sources at a substrate temperature $\mathrm{T}_{\mathrm{s}}$ $=400-800^{\circ} \mathrm{C}$ or depositing at $\mathrm{T}_{\mathrm{s}}<200^{\circ} \mathrm{C}$ and then annealing at higher temperatures. The relative deposition rates of the sources $(\approx 0.1 \mathrm{~nm} / \mathrm{sec})$ were used to adjust the composition of the films. The total film thicknesses ranged from 6-500 nm. The film structure was studied by x-ray diffraction and atomic force microscopy (AFM). The magnetic properties were measured with both a Quantum Design d.c. magnetometer equipped with a 9 Tesla magnet, and by magnetic force microscopy (MFM).

Shown in Fig. 1(a) and (b) are the x-ray diffraction results for $\approx 400$-nm thick $\mathrm{Co}-\mathrm{Sm}$ films deposited simultaneously onto adjacent $\mathrm{MgO}(100)$ and $\mathrm{MgO}(110)$ substrates, respectively $\left(\mathrm{T}_{\mathrm{s}}=600^{\circ} \mathrm{C}\right.$, the $\mathrm{Co}$ and $\mathrm{Sm}$ sputtering sources are operated at 100 and $24 \mathrm{~W}$, respectively). In Fig. 1a, in addition to the $\mathrm{MgO}$ and $\mathrm{W} a$-axis diffraction peaks, a single $\mathrm{Co}$ Sm diffraction peak is observed at $2 \theta=35.52^{\circ}$ with higher-order reflections at $2 \theta=75.2^{\circ}$ and $132.6^{\circ}$. No other crystalline phases or orientations are observed. Rocking curves about the Co-Sm peaks have a full width at half maximum $\Delta \theta=1.3^{\circ}$ indicative of a high degree of crystalline orientation as in epitaxial growth. Figure $1 \mathrm{~b}$ is for the film grown simultaneously onto a $\mathrm{MgO}(111)$ substrate with a W(110) buffer layer which results in a dramatic change in the diffraction pattern. A series of diffraction peaks are observed which corresponds to a periodicity of $0.819 \mathrm{~nm}$. In this orientation, additional peaks indicate the presence of secondary phases. The rocking curve through the Co-Sm peak again shows a high degree of crystalline orientation $\left(\Delta \theta=1.4^{\circ}\right)$. The oriented growth presently precludes a unique determination of the $\mathrm{Co}-\mathrm{Sm}$ phase since a standard $\theta-2 \theta \times$-ray diffraction scan only probes a single direction in the unit cell. However, from the lattice spacing determined from these scans, the phase can be inferred by assuming (i) that the same phase forms on the two substrate but with different crystallographic orientations, and (ii) that the films prefer to grow in low-index orientations. For the samples shown in Fig. 1, the diffraction peaks are best indexed to the $\mathrm{SmCo}_{3}$ phase (see Fig. 1) which is a rhombohedral $\mathrm{Be}_{3} \mathrm{Nb}$-type structure with lattice parameters $\mathrm{a}=0.5055 \mathrm{~nm}$ and $\mathrm{c}=2.457 \mathrm{~nm}$. The 
lattice parameters for this phase calculated from Fig. 1 are 0.5052 and $2.459 \mathrm{~nm}$, respectively. This phase identification as well as determining the epitaxial orientation of the film with respect to the substrate needs to be confirmed by transmission electron diffraction and in-plane x-ray diffraction. AFM images indicate a rough surface morphology with a rms roughness of $\approx 12 \mathrm{~nm}$ and typical in-plane grain size are $\approx 200 \mathrm{~nm}$ for both orientations.

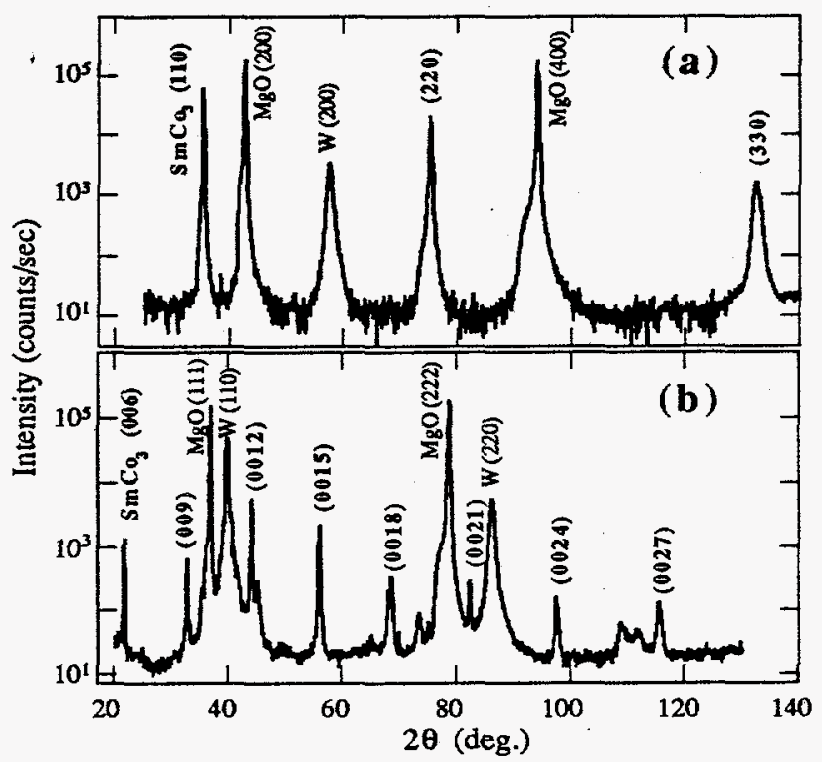

Figure 1: Sm-Co films grown on (a) $\mathrm{MgO}(100)$ and (b) $\mathrm{MgO}(111)$ substrates with W $(100)$ and (110) buffer layers, respectively. The Sm-Co peaks are indexed to the $\mathrm{SmCo}_{3}$ phase.

Shown in Fig. 2 are the magnetic hysteresis loops for the Sm-Co film shown in Fig. 1a. The film has strong in-plane anisotropy requiring $\approx 10 \mathrm{~T}$ to saturate perpendicular to the film and coercive fields $\mathrm{H}_{\mathrm{c}}$ much higher than those typical of sputtered Co-Sm films $[5,6]$. The in-plane coercive field is $3.1 \mathrm{~T}$ at room temperature which increases to $5.1 \mathrm{~T}$ at $5 \mathrm{~K}$ for the $\mathrm{Co}-\mathrm{Sm}$ film on $\mathrm{MgO}(100)$. The $\mathrm{H}_{\mathrm{C}}$ values depend on the in-plane orientation of the $\mathrm{H}$ with respect to the substrate orientation. For $\mathrm{HIIMgO}$ 011]IWW[001] the maximum $\mathrm{H}_{C}=3.1 \mathrm{~T}$ is observed. With HIIMgO[001]|IW[011], lower $\mathrm{H}_{\mathrm{C}}$ values of $2.3 \mathrm{~T}(3.6 \mathrm{~T}$ at $5 \mathrm{~K})$ are observed. The magnetic properties reflect the four-fold symmetry of the substrate and $\mathrm{W}$ buffer layer. The room temperature $\mathrm{H}_{\mathrm{c}}$ values of the $\mathrm{Sm}$-Co film on $\mathrm{MgO}(111)$ is $1.6 \mathrm{~T}$. 


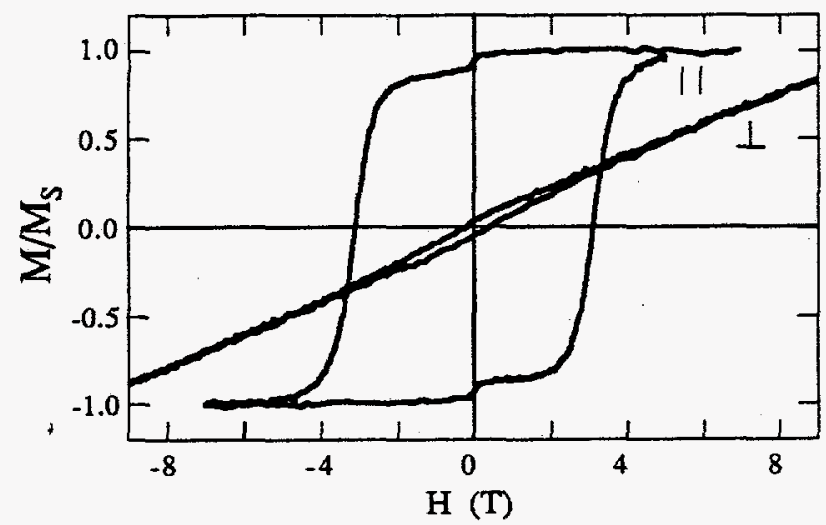

Figure 2: Room-temperature magnetic hysteresis loops for Sm-Co film shown in Fig. 1(a) with the field parallel (II) and perpendicular $(\perp)$ to the film. The in-plane loop is measured with $\mathrm{H} \| \mathrm{MgO}[011]$.

Growth of $\mathrm{Sm}-\mathrm{Fe}$ onto $\mathrm{MgO}(100) / \mathrm{W}(100)$ results in highly-oriented $\left(\Delta \theta \approx 1.1^{\circ}\right)$ single-phase films with a lattice spacing of $0.2402 \mathrm{~nm}$. This spacing is well matched to the (002) reflection of $\mathrm{SmFe}_{12}(0.2403 \mathrm{~nm})$ determined by Wang et al. [7]. AFM images show a smoother growth as compared to Co-Sm films (3-nm rms roughness for a $400-\mathrm{nm}$ film). Growth onto $\mathrm{MgO}(111) / \mathrm{W}(110)$ often results in significant contributions from epitaxial $\alpha-\mathrm{Fe}(110)$.

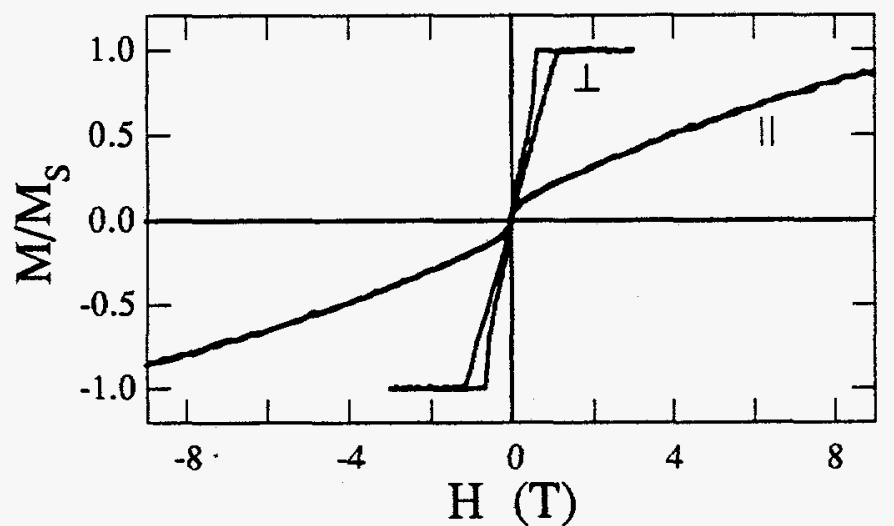

Figure 3: Room-temperature magnetic hysteresis loops for a Sm-Fe film with the field parallel (II) and perpendicular ( $\perp$ ) to the film. The film was grown onto a $\mathrm{MgO}(100)$ substrate.

Shown in Fig. 3 are the magnetization loops for a Sm-Fe film grown onto $\mathrm{MgO}(100)$. There is a strong perpendicular anisotropy. This indicated by 
the sheared in-plane hysteresis loop and is consistent with the $c$-axis anisotropy of $\mathrm{SmFe}_{{ }_{12}}$. The perpendicular anisotropy (estimated by the saturation field of the in-plane loop) is $\approx 10 \mathrm{~T}$ at room temperature and increases to $\approx 27 \mathrm{~T}$ at $5 \mathrm{~K}$. Unlike the Sm-Co film, these films are soft with zero remenance in both orientations suggesting domain formation at low fields. The domain pattern, observed by MFM, is shown in Fig. 4. The magnetization of each contrasted region is either in or out of the plane of the film and the typical stripe width is $\approx 130 \mathrm{~nm}$.

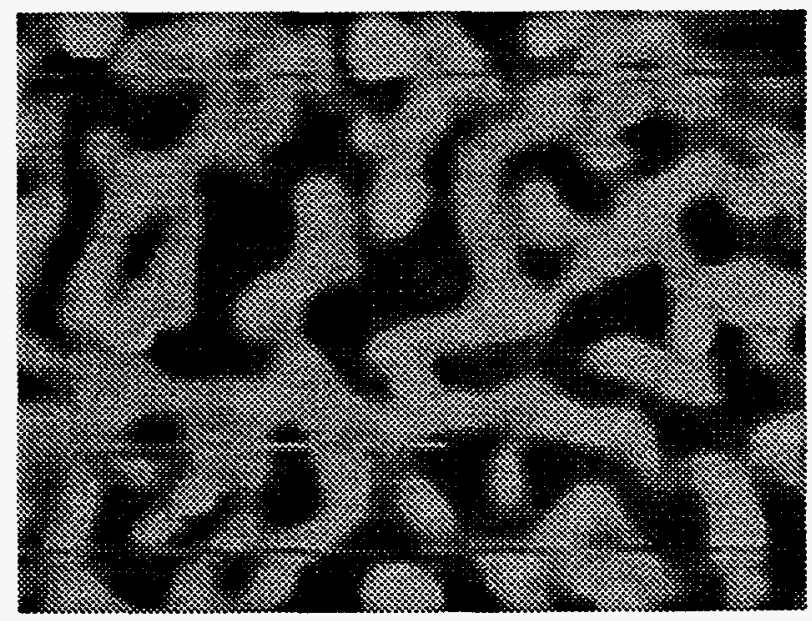

Figure 4: Room-temperature magnetic force microscope image for the $\mathrm{Sm}-\mathrm{Fe}$ film shown in Fig. 3. The horizontal scale of the image is 2 $\mu \mathrm{m}$.

The growth of Nd-Fe films onto W(100) and $\mathrm{W}(110)$ is similar to that of Sm-Co. Using the same indexing procedure outlined for Sm-Co films, the $\mathrm{Nd}-\mathrm{Fe}$ diffraction peaks can be indexed to either the $\mathrm{Nd}_{2} \mathrm{Fe}_{17}$ or $\mathrm{NdFe}_{7}(l 00)$ and $(00 l)$ reflections for the $\mathrm{W}(100)$ and (110) buffer layers, respectively. This latter orientation is consistent with recent reports by Robaut $e$ al. of growth of $c$-axis $\mathrm{Y}_{2} \mathrm{Co}_{17}$ on W(011) by pulsed laser deposition [8]. The calculated lattice parameters are $\mathrm{a}=0.8581 \mathrm{~nm}$ and $\mathrm{c}=1.2482 \mathrm{~nm}$ as compared to the bulk $\mathrm{Nd}_{2} \mathrm{Fe}_{17}$ values of 0.8578 and $1.2462 \mathrm{~nm}$, respectively.

In conclusion, a variety of oriented RE-TM films have been grown onto $\mathrm{MgO}(100)$ and (111) substrates with epitaxial $\mathrm{W}$ buffer layers. The use of epitaxial $W$ buffer layers allows highly oriented single-phase films to be grown. For Sm-Co and Nd$\mathrm{Fe}$ we find $\mathrm{W}(110)$ buffer layers initiate $c$-axis growth and $W(100)$ buffer layers promote growth with the c-axis in-plane. When grown on W(100), 
Sm-Co films have strong in-plane anisotropy and coercivities exceeding $5 \mathrm{~T}$ at $5 \mathrm{~K}$. Sm-Fe films grown on $\mathrm{MgO}(100)$ exhibits perpendicular magnetic anisotropy and are magnetically soft.

1. E. E. Fullerton, M. J. Conover, J. E. Mattson, C. H. Sowers, and S. D. Bader, Phys. Rev. B 48, 15755 (1993).

2. R Skomski and J.M.D. Coey, Phys. Rev. B 48, 15812 (1993).

3. For a recent review see J. J. M. Franse and R. J. Radwanski, in Handbook of Magnetic Materials, Vol. 7, edited by K. H. J. Boschow (Elsevier Science Publishers 1993) p. 307.

4. J. E. Mattson, E. E. Fulleton, C. H. Sowers, and S. D. Bader, J. Vac. Sci. Technol. A 13, 276 (1995).

5. E. M. T. Velu and D. N. Lambeth, J. Appl. Phys. 69, 5175 (1991).

6. Y. Liu, W. B. Robertson, Z. S. Scan, S. Malhotra, M. J. Yu, S. K. Renukunta, S. H. Liou, and D. J. Sellmyer, IEEE Trans. Mag. 30, 4035 (1994).

7. D. Wang, S. H. Liou, P. He, D. J. Sellmyer, G. C. Hadjipanayis, and Y. Zhang, J. Magn. Magn. Mater. 124, 62 (1993).

8. F. Robaut, P. Milkulik, N. Cherief, O. F. K. McGrath, D. Givord, T. Baumbach, J. Y. Beuillen, J. Appl. Phys. 78, 997 (1995). 